\title{
Origin of $\mathrm{Bi}^{3+}$-related luminescence centers in $\mathrm{Lu}_{3} \mathrm{Al}_{5} \mathrm{O}_{12}: \mathrm{Bi}$ and $\mathrm{Y}_{3} \mathrm{Al}_{5} \mathrm{O}_{12}: \mathrm{Bi}$ single crystalline films and the structure of their relaxed excited states
}

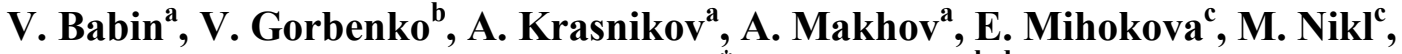 \\ S. Zazubovich ${ }^{\mathrm{a}^{*}}$, Yu. Zorenko ${ }^{\mathrm{b}, \mathrm{d}}$ \\ ${ }^{a}$ Institute of Physics, University of Tartu, Riia 142, 51014 Tartu, Estonia \\ ${ }^{b}$ Ivan Franko National University of Lviv, Gen. Tarnavsky 107, 79017 Lviv, Ukraine \\ ${ }^{c}$ Institute of Physics AS CR, Cukrovarnicka 10, 16253 Prague, Czech Republic \\ ${ }^{d}$ Institute of Physics, Kazimierz Wielki University in Bydgoszcz, \\ Weyssenhoffa sq. 11, 85-090 Bydgoszcz, Poland
}

\begin{abstract}
Single crystalline films ( $\mathrm{SCFs}$ ) of $\mathrm{Y}_{3} \mathrm{Al}_{5} \mathrm{O}_{12}: \mathrm{Bi}$ and $\mathrm{Lu}_{3} \mathrm{Al}_{5} \mathrm{O}_{12}: \mathrm{Bi}$ with different $\mathrm{Bi}^{3+}$ contents are studied at $1.7-300 \mathrm{~K}$ by the time-resolved luminescence spectroscopy methods under excitation in the 2.4-20 eV energy range. Two ultraviolet emission bands of these SCFs, located at 3.99-4.08 eV at $\mathrm{T}<80 \mathrm{~K}$ and at $4.11-4.19 \mathrm{eV}$ at $150 \mathrm{~K}$, arise from the radiative decay of the metastable and radiative minima, respectively, of the triplet relaxed excited state (RES) of a single $\mathrm{Bi}^{3+}$ center, which are related to the ${ }^{3} \mathrm{P}_{0}$ and ${ }^{3} \mathrm{P}_{1}$ levels of a free $\mathrm{Bi}^{3+}$ ion. Their excitation bands located around $4.6 \mathrm{eV}, 5.2 \mathrm{eV}$, and $5.95 \mathrm{eV}$ are assigned to the ${ }^{1} \mathrm{~S}_{0} \rightarrow{ }^{3} \mathrm{P}_{1},{ }^{1} \mathrm{~S}_{0}$ $\rightarrow{ }^{3} \mathrm{P}_{2}$, and ${ }^{1} \mathrm{~S}_{0} \rightarrow{ }^{1} \mathrm{P}_{1}$ transitions, respectively, in free $\mathrm{Bi}^{3+}$ ions. The luminescence of dimer $\mathrm{Bi}^{3+}-\mathrm{Bi}^{3+}$ centers is not detected in the SCFs studied. The lower-energy $(\approx 2.6 \mathrm{eV})$ visible emission of these SCFs is due to an exciton, localized near a single $\mathrm{Bi}^{3+}$ ion, while the higherenergy $(2.75 \mathrm{eV})$ visible emission, an exciton, localized near a dimer $\mathrm{Bi}^{3+}-\mathrm{Bi}^{3+}$ center. Temperature dependences of the luminescence decay times are measured for the ultraviolet and visible emissions in the 1.7-300 $\mathrm{K}$ temperature range and simulated by phenomenological models of the dynamics of corresponding excited states. The quantitative parameters of RESs (the energy separations between the energy levels and the rates of the radiative and nonradiative transitions from these levels) are calculated.
\end{abstract}

PACS: 78.55 Hx; 78.47.+p; 71.55.Ht; 71.35-y

\section{Introduction}

$\mathrm{Bi}^{3+}$-doped rare-earth aluminate and gallate garnets, where a $\mathrm{Bi}^{3+}$ ion substitutes for a trivalent rare-earth ion, can be considered as perspective materials for scintillators due to an intense and fast $\mathrm{Bi}^{3+}$-related emission [1-3]. In SCFs of $\mathrm{Bi}^{3+}$-doped aluminum garnets prepared by the liquid phase epitaxy (LPE) method, a large and variable concentration of $\mathrm{Bi}^{3+}$ ions can be achieved [3]. However, the luminescence of $\mathrm{Bi}^{3+}$ centers in garnets has not been extensively studied. In the emission spectrum of $\mathrm{Y}_{3} \mathrm{Ga}_{5} \mathrm{O}_{12}$ : $\mathrm{Bi}[1,4], \mathrm{Gd}_{3} \mathrm{Ga}_{5} \mathrm{O}_{12}: \mathrm{Bi}$ [4], $\mathrm{Y}_{3} \mathrm{Al}_{5} \mathrm{O}_{12}: \mathrm{Bi}$ [2-4], and $\mathrm{Lu}_{3} \mathrm{Al}_{5} \mathrm{O}_{12}: \mathrm{Bi}$ [3-4], two bands, located in the ultraviolet (UV) and in the visible (VIS) spectral range, were observed. The UV emission was assigned to the radiative decay of the triplet RES of $\mathrm{Bi}^{3+}$ centers. Two components of this emission were connected with the electronic transitions, corresponding to the ${ }^{3} \mathrm{P}_{1} \rightarrow{ }^{1} \mathrm{~S}_{0}$ and ${ }^{3} \mathrm{P}_{0} \rightarrow{ }^{1} \mathrm{~S}_{0}$ transitions of a free $\mathrm{Bi}^{3+}$ ion [1]. The VIS emission was assigned to the $\mathrm{Bi}^{3+}$-related bound excitons [5] or to complex $\mathrm{Bi}^{3+}$-based centers [2-4]. Recently, we have carried out the study of the emission and excitation spectra and the luminescence decay kinetics of $\mathrm{Bi}^{3+}$-related centers in SCFs of $\mathrm{Lu}_{3} \mathrm{Al}_{5} \mathrm{O}_{12}$ : $\mathrm{Bi}$ (LuAG:Bi) [6] and $\mathrm{Y}_{3} \mathrm{Al}_{5} \mathrm{O}_{12}: \mathrm{Bi}(\mathrm{YAG}: \mathrm{Bi}$ ) [7] at 4.2- $400 \mathrm{~K}$. It was found that their emission 
spectrum consists of two types of bands with much different properties. Two UV emission bands were assigned to the decay of the radiative and metastable minima of the triplet RES of a $\mathrm{Bi}^{3+}$ centre. The radiative decay of an exciton, localized near a single $\mathrm{Bi}^{3+}$ ion, was suggested to result in the appearance of the lower-energy $(\approx 2.6 \mathrm{eV})$ VIS emission band. The weaker higherenergy VIS emission band $(2.75 \mathrm{eV})$, showing a stronger intensity dependence on the $\mathrm{Bi}^{3+}$ content, was assumed to arise from an exciton localized near a dimer $\mathrm{Bi}^{3+}-\mathrm{Bi}^{3+}$ center. Nevertheless, to confirm the hypothesis on the excitonic origin of VIS emissions and to define the structure of the corresponding RESs in both qualitative and quantitative way, the detailed study of the luminescence decay kinetics at low temperatures is needed.

Despite the suggestion that the $2.75 \mathrm{eV}$ emission is connected with the dimer $\mathrm{Bi}^{3+}-\mathrm{Bi}^{3+}$ centers, no luminescence from such centers was found in [6-7]. However, later [8] it was concluded that the $3.995 \mathrm{eV}$ emission band and the corresponding very narrow $4.32 \mathrm{eV}$ excitation band of YAG:Bi arise from the dimer centers.

As a result, in the present work we focus on both the detailed investigation of the luminescence characteristics of LuAG:Bi and YAG:Bi SCFs in a wide temperature range down to $1.7 \mathrm{~K}$ as well as a thorough search for the dimer $\mathrm{Bi}^{3+}-\mathrm{Bi}^{3+}$ centers. We aim to obtain additional information on the origin of the related luminescence centers as well as on the structure and quantitative characteristics of the RESs responsible for their luminescence.

\section{Experimental details}

SCFs of LuAG:Bi and YAG:Bi were grown by the LPE method from the melt-solution based on a $\mathrm{Bi}_{2} \mathrm{O}_{3}$ oxide flux [3]. Three samples of LuAG:Bi with $0.07,0.183$, and 1.34 at $\%$ of $\mathrm{Bi}^{3+}$ and two samples of YAG:Bi with 0.133 and 0.92 at $\%$ of $\mathrm{Bi}^{3+}$ were studied. Single crystals of YAG were used as the substrates. The concentration of $\mathrm{Bi}^{3+}$ ions was determined with a JEOL JXA-733 electron microprobe analyser.

The steady-state emission and excitation spectra of the LuAG:Bi and YAG:Bi SCFs and the YAG substrate were measured at the same conditions at 80-350 K under excitation in the 2.4-6.2 eV energy range. The set-up consisted of a deuterium DDS-400 lamp, two monochromators (SF-4 and SPM-1) and a photomultiplier (FEU-39 or FEU-79) with an amplifier and recorder. The spectra were corrected for the spectral distribution of the excitation light, the transmission and dispersion of the monochromators and the spectral sensitivity of the detectors.

Luminescence decay kinetics in the $\mu \mathrm{s}-\mathrm{ms}$ time range was measured at $1.7-300 \mathrm{~K}$ by the same set-up under excitation with a xenon flash lamp FX-1152 (EG\&G) (the pulse duration of about $1 \mu \mathrm{s}$ and maximum repetition frequency of $300 \mathrm{~Hz}$ ). The decay curves $\mathrm{I}(\mathrm{t})$ were measured at the same conditions for different emission and excitation energies. This allows us to obtain the time-resolved emission and excitation spectra at any moment of time ( $\mathrm{t}$ ) after the excitation pulse. The decay kinetics was also measured by a modified Spectrofluorometer 199S (Edinburgh Instruments) under excitation with a nanosecond coaxial hydrogen-filled flashlamp (IBH Scotland) and using two single grating monochromators. The detection was performed by an IBH-04 photomultiplier module using the method of time-correlated single photon counting. A deconvolution procedure (SpectraSolve software package) was applied to extract true decay times using the multiexponential approximation. The time-resolved excitation spectra in the 4$20 \mathrm{eV}$ energy range were measured at $10 \mathrm{~K}$ under synchrotron excitation at SUPERLUMI station (HASYLAB at DESY, Hamburg, Germany). The experiments at low temperatures were carried out with the use of an immersion helium cryostat, close-cycle refrigerator or vacuum nitrogen cryostat. The temperatures in the 1.7-4.2 $\mathrm{K}$ range were achieved by pumping of the He vapour from the immersion helium cryostat. 


\section{Experimental results and discussion}

\subsection{The low-temperature luminescence decay kinetics and the quantitative characteristics of the corresponding relaxed excited states}

As previously observed, the decay time of both VIS emissions in LuAG:Bi and YAG:Bi increases as the temperature decreases down to $4.2 \mathrm{~K}$ [6-7]. Therefore, to more reliably determine the quantitative characteristics of the corresponding RESs, the investigated temperature range was extended down to $1.7 \mathrm{~K}$. Temperature dependences of the decay times of the UV and VIS emissions of LuAG:Bi and YAG:Bi are shown in Figs. 1 and 2, respectively (full circles are experimental data). In the decay kinetics of the UV emissions of LuAG:Bi and YAG:Bi, located at low temperatures at $4.08 \mathrm{eV}$ and $3.99 \mathrm{eV}$, respectively (see Fig. 3a, curves 1, 1'), one observes mainly a slow component. The intensity of the fast component reported in [6-7] is negligible, and its very short decay time ( $0.1-0.6 \mathrm{~ns})$ origin of a very weak fast (0.1-0.6 ns) component reported in [6-8] is not clear and needs further investigations. indicates that it cannot arise from the spin forbidden transitions of the triplet state of $\mathrm{Bi}^{3+}$. Probably, this component arises from the scattered synchrotron radiation. At $\mathrm{T}<80 \mathrm{~K}$, the slow component decay time is constant $(\tau \sim 1 \mathrm{~ms})$ and at $\mathrm{T}>100 \mathrm{~K}$, it exponentially decreases with increasing temperature (Figs. 1a and 2a).

Thus, the decay kinetics of the UV emissions of LuAG:Bi and YAG:Bi is similar to that observed for the triplet emission of $\mathrm{Bi}^{3+}$ centers in some other hosts (see, e.g., [1,9-11]). It can be described within the phenomenological model used in [1] for $\mathrm{Bi}^{3+}$ centers in $\mathrm{Y}_{3} \mathrm{Ga}_{5} \mathrm{O}_{12}$. The model is sketched in Fig. 4a. The time evolution of the populations $N_{1}, N_{2}$ of the excited levels 1 and 2, respectively, can be described by the following rate equations:

$$
\mathrm{d} N_{1} / \mathrm{d} t=-k_{1} N_{1}-k_{12} N_{1}+k_{21} N_{2}-k_{1 x} N_{1}
$$

$$
\mathrm{d} N_{2} / \mathrm{d} t=-k_{2} N_{2}-k_{21} N_{2}+k_{12} N_{1}-k_{2 x} N_{2} \text {, }
$$

where $k_{1}, k_{2}, k_{12}, k_{21}$, and $k_{1(2) x}$ are radiative transition rates from levels 1,2 , non-radiative rates of phonon assisted transitions between the radiative level 2 and metastable level 1 and the quenching channel from the level 1(2), respectively. Non-radiative transitions between levels 1,2 can be written as:

$$
k_{21}=K(n+1), k_{12}=K n, \quad n=1 /\left[\exp \left(D / k_{B} T\right)-1\right] .
$$

Here $K, n, D$ are the zero-temperature transition rate between the levels 1 and 2, the BoseEinstein factor and energy spacing between the levels, respectively. Non-radiative quenching channel is considered in the usual barrier form:

$$
k_{1(2) x}=K_{1(2) x} \exp \left(-E_{1 x} / k_{B} T\right)
$$

with $K_{1 x}$ being a frequency factor and $E_{1 x}$ the height of the barrier. In the case of the UV emissions no quenching channels are considered.

The best fit of the experimental $\tau(\mathrm{T})$ dependence is shown as a solid line in Figs. 1a and 2a for LuAG:Bi and YAG:Bi, respectively. The parameters of the triplet RES corresponding to the best fit are also reported in the figures. The obtained data indicate that at $\mathrm{T}<80 \mathrm{~K}$, the contribution to the observed radiative decay comes only from the metastable minima (level 1) of the triplet RES of $\mathrm{Bi}^{3+}$. At $\mathrm{T}>100 \mathrm{~K}$, the shortening of the UV emission decay time and the high-energy shift of the UV emission band with increasing temperature are caused by thermally stimulated non-radiative transitions between the metastable (level 1) and radiative (level 2) minima of the triplet RES.

As was already shown in [6-7], the decay kinetics of the visible emissions of LuAG:Bi and YAG:Bi are rather complicated. In the present work, it was found that at $\mathrm{T}<4.2 \mathrm{~K}$, the decay times of the VIS emissions remain constant. At $1.7 \mathrm{~K}$, the decay time of the most intense component of the $\approx 2.6 \mathrm{eV}$ emission is about $38 \mu \mathrm{s}$ in YAG:Bi and $26 \mu \mathrm{s}$ in LuAG:Bi (Figs. $1 \mathrm{~b}$ 
and $2 \mathrm{~b}$ ). For the $2.75 \mathrm{eV}$ emission, the corresponding decay times are $33 \mu \mathrm{s}$ and $40 \mu \mathrm{s}$, respectively (Figs. 1c and 2c). The temperature dependence of the decay time of each VIS band is similar in both SCFs. As the temperature increases, the decay time of the $2.75 \mathrm{eV}$ emission decreases down to $4-5 \mu$ s at about $50 \mathrm{~K}$. The shortening of its decay time at $\mathrm{T}>170 \mathrm{~K}$ is due to the thermal quenching of this emission [6-7]. As the temperature increases, the decay time of the $\approx 2.6 \mathrm{eV}$ emission decreases up $10 \mathrm{~K}$, then remains almost constant, and at $\mathrm{T}>25 \mathrm{~K}$ it decreases again. No thermal quenching of this emission takes place at $\mathrm{T}<100 \mathrm{~K}$ [6-7]. Thus, the shortening of the decay time has to be caused by a thermally assisted population of a higher energy level.

According to the hypothesis about the excitonic origin of both VIS emissions we attempted to describe their decay kinetics within the phenomenological model similar to that described above, where the excited state levels 1 and 2 would correspond to those of localized exciton triplet states. In the case of higher-energy VIS emissions we employed the thermal quenching from both excited states (see the scheme in Fig. $4 \mathrm{~b}$ ). In the case of the $\approx 2.6 \mathrm{eV}$ emission we considered an extended model taking into account the additional transitions to and from the singlet level 3 of the exciton state lying above the triplet levels of the RES. The model is sketched in Fig $4 \mathrm{c}$ and the rate equations now read:

$$
\begin{aligned}
& \mathrm{d} N_{1} / \mathrm{d} t=-k_{1} N_{1}-k_{12} N_{1}-k_{13} N_{1}+k_{21} N_{2}+k_{31} N_{3} \\
& \mathrm{~d} N_{2} / \mathrm{d} t=-k_{2} N_{2}-k_{21} N_{2}-k_{23} N_{2}+k_{12} N_{1}+k_{32} N_{3} \\
& \mathrm{~d} N_{3} / \mathrm{d} t=-k_{3} N_{3}-k_{31} N_{3}-k_{32} N_{3}+k_{13} N_{1}+k_{23} N_{2},
\end{aligned}
$$

where analogous parameters have the same meaning as in (1). In addition, $k_{3}$ is the radiative transition rate from the singlet level 3 and $k_{3(2) 1}, k_{1(2) 3}$ are non-radiative rates of transitions between the levels 3(2) and 1:

$$
\text { Since } \mathrm{D}<<\mathrm{E} \text { we consider }
$$

$$
k_{31}=K^{\prime}\left(n^{\prime}+1\right), k_{13}=K^{\prime} n^{\prime}, n^{\prime}=1 /\left[\exp \left(E / k_{B} T\right)-1\right] .
$$

$$
k_{31}=k_{32}, k_{13}=k_{23}
$$

The best fit of the experimental $\tau(\mathrm{T})$ dependence is displayed as a solid line in Figs. 1b,c and 2 b,c. The parameters used to obtain the best fit are reported in the figures. The model described above successfully reproduces the data and the considered RES structure as well as parameters obtained are characteristic for the localized exciton states in aluminum garnets (see, e.g., [12]). Thus, the results of our decay kinetics modelling strongly support the hypothesis on the excitonic origin of both VIS emissions in LuAG:Bi and YAG:Bi made in [6-7]. At T<4.2 $\mathrm{K}$, the visible emissions are due to the radiative decay of the metastable minima of the triplet state of the localized exciton. The shortening of their decay time with increasing temperature in the $4.2-50 \mathrm{~K}$ range is caused by thermally stimulated non-radiative transitions between the metastable and radiative minima of the triplet RES. The energy distance between them is about 2-4 meV. Around $50 \mathrm{~K}$, the thermal equilibrium between these minima is reached. The shortening of the decay time at further increase of the temperature in the case of the $2.75 \mathrm{eV}$ emission is caused by thermal quenching of this emission at $\mathrm{T}>100 \mathrm{~K}[6,7]$ with the activation energies of about 100 and $200 \mathrm{meV}$, but in the case of the $\approx 2.6 \mathrm{eV}$ emission, which is not thermally quenched up to $160-175 \mathrm{~K}$ [6-7], by thermally stimulated transitions between the triplet and singlet localized exciton states separated by the energy distance of 100-150 meV.

For $\mathrm{Tl}^{+}$and $\mathrm{Pb}^{2+}$-doped caesium halides, the appearance of the localized exciton states under excitation in the impurity-induced absorption bands was considered as a result of the electron transfer from the neighbouring halide ion perturbed by an impurity ion. As a result, a mobile hole in the valence band and the electron impurity center were created. A fast tunnelling recombination of the self-trapped holes and electrons close to the impurity ion results in the formation of the localized exciton state and its further radiative decay (see, e.g., [13]). In some cases, stable electron and hole centers were also optically created in the crystal 
lattice and detected in the TSL and EPR studies of the UV-irradiated crystals [13-15]. However, after the UV irradiation of LuAG:Bi and YAG:Bi SCFs in the 4.5-6.0 eV energy range at $80-300 \mathrm{~K}$, no TSL is observed. It means that the fast radiative recombination of the optically created electron and hole centers considerably dominates in the SCFs studied.

Taking into account the positions of energy levels of the free $\mathrm{Bi}^{3+}$ ion and the position of the lowest-energy $\mathrm{A}$ absorption band, corresponding to the ${ }^{1} \mathrm{~S}_{0} \rightarrow{ }^{3} \mathrm{P}_{1}$ transition $(4.63 \mathrm{eV}$ in LuAG), the positions of the $\mathrm{B}$ and $\mathrm{C}$ bands of the $\mathrm{Bi}^{3+}$ center in LuAG:Bi were estimated in [6]. It was concluded that the $\mathrm{C}$ band, arising from the ${ }^{1} \mathrm{~S}_{0} \rightarrow{ }^{1} \mathrm{P}_{1}$ transitions, should be located at $\approx 5.95 \mathrm{eV}$ and the $\mathrm{B}$ absorption band, at about $5.2 \mathrm{eV}$. In Fig. 5 we show the excitation spectrum of the UV emission of LuAG:Bi measured at $10 \mathrm{~K}$. As it is evident from the inset, a strong band near $6 \mathrm{eV}$ is really present in the excitation spectrum of the $\mathrm{UV}$ emission on the slope of the intense bands arising from the excitons perturbed by $\mathrm{Bi}^{3+}$ ions [12]. Similar consideration for $\mathrm{YAG}: \mathrm{Bi}$, where the $\mathrm{A}$ band is located at $4.57 \mathrm{eV}$, allows the conclusion that the $\mathrm{C}$ and $\mathrm{B}$ bands should be located at about $5.9 \mathrm{eV}$ and $5.15 \mathrm{eV}$.

\subsection{The search for dimer $B i^{3+}-B i^{3+}$ centers in LuAG:Bi and YAG:Bi}

The studies of $\mathrm{ns}^{2}$-ions-doped alkali halides (see, e.g., [16,17]) and aluminum perovskites and garnets [18] showed that the spectral bands of dimer impurity centers are usually noticeably shifted to lower-energies with respect to the bands of the corresponding single centers. Furthermore, their luminescence decay time is shorter.

The A absorption band of $\mathrm{Bi}^{3+}$ centers in $\mathrm{LuAG}: \mathrm{Bi}$ and YAG:Bi measured at $\mathrm{RT}$ is shown in Figs. $6 \mathrm{a}$ and $7 \mathrm{a}$, respectively. To detect the presence of dimer $\mathrm{Bi}^{3+}-\mathrm{Bi}^{3+}$ centers, the UV emission spectra of LuAG:Bi and YAG:Bi SCFs with different Bi contents were measured at different temperatures with narrow slits under different excitations in the A absorption region. The dependences of both the UV emission band position and FWHM on the excitation energy are shown in Figs. 6b,c for LuAG:Bi and Figs. 7b,c for YAG:Bi. It is evident that under excitation in the 4.30-4.85 eV energy range, the position and FWHM of the emission band are independent of the excitation energy. The UV emission decay time does not depend on the excitation and emission energy either (Fig. 8). The same results are obtained for the SCFs with different $\mathrm{Bi}$ contents. Therefore, in this energy range, there is no evidence of the presence of dimer $\mathrm{Bi}^{3+}$ centers. A very small lower-energy shift of the emission band and an increase of the FWHM are observed only under $4.25 \mathrm{eV}$ excitation in YAG:Bi and $4.3 \mathrm{eV}$ excitation in LuAG:Bi. However, as it will be shown below, the observed effects arise not from $\mathrm{Bi}^{3+}-\mathrm{Bi}^{3+}$ centers but from the overlap of the $\mathrm{Bi}^{3+}$ emission with the emission of the YAG substrate used for the preparation of the SCFs studied.

Indeed, the study at the same experimental conditions of luminescence characteristics of the YAG substrate shows that under excitation in the 4.2-4.4 eV energy range, the bands at $3.96 \mathrm{eV}$ and $\approx 3.3 \mathrm{eV}$ are observed in its emission spectrum (Fig. $3 b$, curve 1). The $3.96 \mathrm{eV}$ band is strongly overlapped with the UV emission band of the single $\mathrm{Bi}^{3+}$ centers. The main excitation band of the $3.96 \mathrm{eV}$ emission, peaking at $4.35 \mathrm{eV}$, is located just at the low-energy side of the A absorption band (curve 2). Under excitation in the energy range around $4.3 \mathrm{eV}$ where the absorption of $\mathrm{Bi}^{3+}$ centers at low temperatures is relatively small, the emission intensities of the substrate and the SCFs are comparable. Therefore, we conclude that $\mathrm{Bi}^{3+}-\mathrm{Bi}^{3+}$ centers do not appear in the luminescence of the SCFs studied. Probably, the formation of the exciton, localized near these centers, is much preferable process as compared with the radiative decay of the $\mathrm{Bi}^{3+}-\mathrm{Bi}^{3+}$ centers. Similar effect was observed, e.g., in CsI:Tl, where the strong yellow emission of an exciton localized near a $\mathrm{Tl}^{+}$ion considerably prevails over the much weaker UV emission of $\mathrm{Tl}^{+}$centers [19]. 
The appearance of a narrow $4.32 \mathrm{eV}$ band in the excitation spectrum of the UV emission, ascribed in [8] to dimer $\mathrm{Bi}^{3+}$ centers, can be explained in the following way. From the absorption spectra of $\mathrm{Bi}^{3+}$ centers in LuAG:Bi and YAG:Bi shown in Figs. 6a and 7a it is evident that in the $\mathrm{A}$ absorption band maximum $(\approx 4.6 \mathrm{eV})$, the optical density is $\mathrm{OD}>>3$. In case of small optical density $(\mathrm{OD}<0.5)$ the absorption and excitation spectra should coincide (see, e.g. [1]), but in case of large optical density (OD>2), the luminescence intensity should not depend on the excitation energy in the whole range of the strong absorption. The appearance in the excitation spectrum of the UV emission of narrow peaks at both the highenergy and the low-energy sides of the A absorption band (like those observed in [8] at $4.7 \mathrm{eV}$ and $4.32 \mathrm{eV}$ ) as well as "a hole" in the region of the maximum absorption are caused by the well-known technical effect, namely, by the peculiarities of the exciting light reflection and scattering caused by a huge absorption in this energy range in the SCFs studied. Relative intensities of the above-mentioned narrow peaks and the depth of the "hole" strongly depend on a sample position and its illumination conditions (compare, e.g., curves 2 and 2' in Fig. 3a and the excitation spectra of YAG:Bi reported in [7] and [8]). The positions of these peaks also depend on optical density, i.e., on $\mathrm{Bi}^{3+}$ concentration. Just these effects are responsible for the distorted structure of the A excitation band of $\mathrm{Bi}^{3+}$ centers in LuAG:Bi and YAG:Bi SCFs observed in [6-8] (as well as in the present paper, see, e.g., Figs. 3a and 8).

\section{Conclusions}

The detailed study of the decay kinetics of the UV and VIS emission bands in SCFs of LuAG:Bi and YAG:Bi in a wide temperature range down to $1.7 \mathrm{~K}$ strongly supports the conclusions made in [6-7]. In particular, the UV emission arises from the decay of the metastable and radiative minima of the triplet $\mathrm{RES}$ of $\mathrm{Bi}^{3+}$ center, while the lower-energy and the higher-energy VIS emission arise from the radiative decay of the excitons localized near single and dimer $\mathrm{Bi}^{3+}$ centers, respectively. The excitation bands of the $\mathrm{UV}$ emission, located around $4.6 \mathrm{eV}, 5.2 \mathrm{eV}$ and $5.95 \mathrm{eV}$, are assigned to the ${ }^{1} \mathrm{~S}_{0} \rightarrow{ }^{3} \mathrm{P}_{1},{ }^{1} \mathrm{~S}_{0} \rightarrow{ }^{3} \mathrm{P}_{2}$, and ${ }^{1} \mathrm{~S}_{0} \rightarrow{ }^{1} \mathrm{P}_{1}$ transitions of a free $\mathrm{Bi}^{3+}$ ion, respectively. We proposed the phenomenological models to describe the excited-state dynamics of all the luminescence centers studied. Application of these two- or three-excited-level models on the temperature evolution of the luminescence decay times allowed determination of characteristic parameters of the corresponding relaxed excited states (the energy separations between the excited states and the rates of the radiative and non-radiative transitions). Despite the existence of the excitons localized near dimer $\mathrm{Bi}^{3+}$ $\mathrm{Bi}^{3+}$ centers, no luminescence from such centers is found. We suggest that the formation of the excitons localized near these centers is-much preferable process comparing to the radiative decay of the $\mathrm{Bi}^{3+}-\mathrm{Bi}^{3+}$ centers themselves.

\section{Acknowledgements}

The work was supported by the projects of the Estonian (No. 8678), Czech (No. 202/08/0893 and GA AV IAA100100810), and Ukrainian (No. SF-28F) Science Foundations, as well as by the II-20052049 EC project of DESY, Hamburg and NATO CBP.NUKR.CLG984305.

\section{References}


1. M. Nikl, A. Novoselov, E. Mihokova, K. Polak, M. Dusek, B. McClune, A. Yoshikawa, T. Fukuda, J. Phys.: Condens. Matter 17, 3367 (2005).

2. Yu. Zorenko, V. Gorbenko, T. Voznyak, V. Vistovsky, S. Nedilko, M. Nikl, Radiat. Meas. 42, 882 (2007).

3. Yu. Zorenko, J.A. Mares, R. Kucerkova, V. Gorbenko, V. Savchun, T. Voznyak, M. Nik1, A. Beitlerova, K. Jurek, J. Phys. D: Appl. Phys. 42, 075501 (2009).

4. A.A. Setlur, A.M. Srivastava, Optical Materials 29, 410 (2006).

5. M. Ilmer, B.C. Grabmaier, G. Blasse, Chem. Mater. 6, 204 (1994).

6. V. Babin, V. Gorbenko, A. Krasnikov, A. Makhov, M. Nikl, K. Polak, S. Zazubovich,Yu. Zorenko, J. Phys.: Condens. Matter 21, 415502 (2009).

7. V. Babin, V. Gorbenko, A. Krasnikov, A. Makhov, M. Nikl, S. Zazubovich, Yu. Zorenko, Radiat. Meas. 45, 331 (2010).

8. Yu. Zorenko, V. Gorbenko, T. Voznyak, V. Jary, M. Nikl, J. Lumin. 130, 1963 (2010).

9. A. Wolfert, G. Blasse, J. Lumin. 33, 213 (1985).

10. R. Aceves, M. Barboza Flores, A. Maaroos, V. Nagirnyi, R. Perez Salas, T. Tsuboi, S. Zazubovich, V. Zepelin, Phys. Stat. Sol. B 194, 619 (1996).

11. H. Donker, N. Yamashita, W.M.A. Smit, G. Blasse, Phys. Stat. Sol. B 156, 537 (1989).

12. V. Babin, V. Bichevin, V. Gorbenko, M. Kink, A. Makhov, Yu. Maksimov, M. Nikl, G. Stryganyuk, S. Zazubovich, Yu. Zorenko, Phys. Stat. Sol. B 248, 1505 (2011).

13. V. Babin, K. Kalder, A. Krasnikov, S. Zazubovich, J. Lumin. 96, 75 (2002).

14. S. Zazubovich, R. Aceves, M. Barboza Flores, P. Fabeni, T. Kärner G.P. Pazzi, R. Perez Salas, N. Jaanson, J. Phys.: Condens. Matter 9, 7249 (1997).

15. V. Babin, A. Krasnikov, S. Zazubovich, Rad. Eff. Def. Solids 158, 227 (2003).

16. V. Nagirnyi, T. Soovik, P. Vaino, S. Zazubovich, N. Jaanson, Phys. Stat. Sol. B 164, 493 (1991).

17. T. Tsuboi, P.W.M. Jacobs, J. Phys. Chem. Sol. 52, 69 (1991).

18. V. Babin, V. Bichevin, V. Gorbenko, A. Makhov, E. Mihokova, M. Nikl, A. Vedda, S. Zazubovich, Yu. Zorenko, Phys. Stat. Sol. B 246, 1318 (2009).

19. V. Nagirnyi, S. Zazubovich, V. Zepelin, M. Nikl, G.P. Pazzi, Chem. Phys. Letters 227, 533 (1994).

\section{Figure captions}

Fig. 1. Temperature dependences of the decay times of (a) the UV emission, (b) the $2.6 \mathrm{eV}$ emission, and (c) the $2.75 \mathrm{eV}$ emission of LuAG:Bi SCFs. Solid circles are experimental data. Solid lines are best fits of the two $(a, b)$ or three (c) excited-state level models shown in Fig. 4 to the experimental data, (for details see the text). The parameters of the fit are reported in the figures. (a) $\mathrm{E}_{\mathrm{exc}}=4.6 \mathrm{eV}, \mathrm{E}_{\mathrm{em}}=4.1 \mathrm{eV}$; (b) $\mathrm{E}_{\mathrm{exc}}=4.7 \mathrm{eV}, \mathrm{E}_{\mathrm{em}}=2.4 \mathrm{eV}$; (c) $\mathrm{E}_{\mathrm{exc}}=5.3 \mathrm{eV}, \mathrm{E}_{\mathrm{em}}=3.2$ $\mathrm{eV}$.

Fig. 2. Temperature dependences of the decay times of (a) the UV emission, (b) the 2.63 $\mathrm{eV}$ emission, and (c) the $2.75 \mathrm{eV}$ emission of YAG:Bi SCFs. Solid circles are experimental data. Solid lines are best fits of the two $(a, b)$ or three (c) excited-state level models shown in Fig. 4 to the experimental data, (for details see the text). The parameters of the fit are reported in the figures. (a) $\mathrm{E}_{\mathrm{exc}}=4.55 \mathrm{eV}, \mathrm{E}_{\mathrm{em}}=4.0 \mathrm{eV}$; (b) $\mathrm{E}_{\mathrm{exc}}=4.5 \mathrm{eV}, \mathrm{E}_{\mathrm{em}}=2.4 \mathrm{eV}$; (c) $\mathrm{E}_{\mathrm{exc}}=5.3 \mathrm{eV}$, $\mathrm{E}_{\mathrm{em}}=3.1 \mathrm{eV}$.

Fig. 3. (a) The UV emission bands (curves 1, 1') and the corresponding A excitation bands (curves 2, 2', 2") of $\mathrm{Bi}^{3+}$ centers in LuAG:Bi (curves 1, 2, 2') and YAG:Bi (curves 1', 2'). Curves 2 and 2' are measured at slightly different crystal positions. (b) Emission spectrum 
$\left(E_{\mathrm{exc}}=4.25 \mathrm{eV}\right)\left(\right.$ curve 1) and excitation spectra for $\mathrm{E}_{\mathrm{em}}=3.85 \mathrm{eV}$ (curve 2) and $\mathrm{E}_{\mathrm{em}}=3.2 \mathrm{eV}$ (curve 2') measured for the YAG substrate. $\mathrm{T}=80 \mathrm{~K}$.

Fig. 4. Energy level diagrams used for the description of the excited states dynamics of the luminescence centers responsible for (a) the UV emission, (b) the $2.75 \mathrm{eV}$ emission, and (c) the the $\approx 2.6 \mathrm{eV}$ emission of $\mathrm{Bi}^{3+}$-doped aluminum garnets. For details, see the text.

Fig. 5. Time-resolved excitation spectra measured at $10 \mathrm{~K}$ for the $\mathrm{UV}$ emission of $\mathrm{Bi}^{3+}$ centers in LuAG:Bi SCF. The time windows are 2-10 ns and 97-150 ns after the excitation pulse. In the inset, the integral spectrum in the 5.6-6.8 eV range is shown in more detail.

Fig. 6. (a) Absorption spectrum of LuAG:Bi SCF at 295 K. Dependences of the (b) FWHM and (c) maximum position of the UV emission band of LuAG:Bi SCF on the excitation energy measured at $4.2 \mathrm{~K}$ (empty circles), $80 \mathrm{~K}$ (triangles), and $295 \mathrm{~K}$ (solid circles). Spectral width of the slit for the excitation was $5 \mathrm{~nm}$, and for the emission, $5 \mathrm{~nm}$ at $295 \mathrm{~K}$ and $3.5 \mathrm{~nm}$ at $4.2 \mathrm{~K}$.

Fig. 7. (a) Absorption spectrum of YAG:Bi SCF at 295 K. Dependences of the (b) FWHM and (c) maximum position of the UV emission band of YAG:Bi SCF on the excitation energy measured at $4.2 \mathrm{~K}$ (empty circles) and $295 \mathrm{~K}$ (solid circles). Spectral width of the slit for the excitation was $5 \mathrm{~nm}$, and for the emission, $5 \mathrm{~nm}$ at $295 \mathrm{~K}$ and $3.5 \mathrm{~nm}$ at $4.2 \mathrm{~K}$.

Fig. 8. Time-resolved UV emission (empty circles) and excitation (solid circles) spectra of (a) LuAG:Bi SCF and (b) YAG:Bi SCF and dependences of the decay time of the UV emission on the excitation energy and on the emission energy at $\mathrm{E}_{\mathrm{exc}}=4.35-4.4 \mathrm{eV}$ (triangles). $\mathrm{T}=4.2 \mathrm{~K}$. 


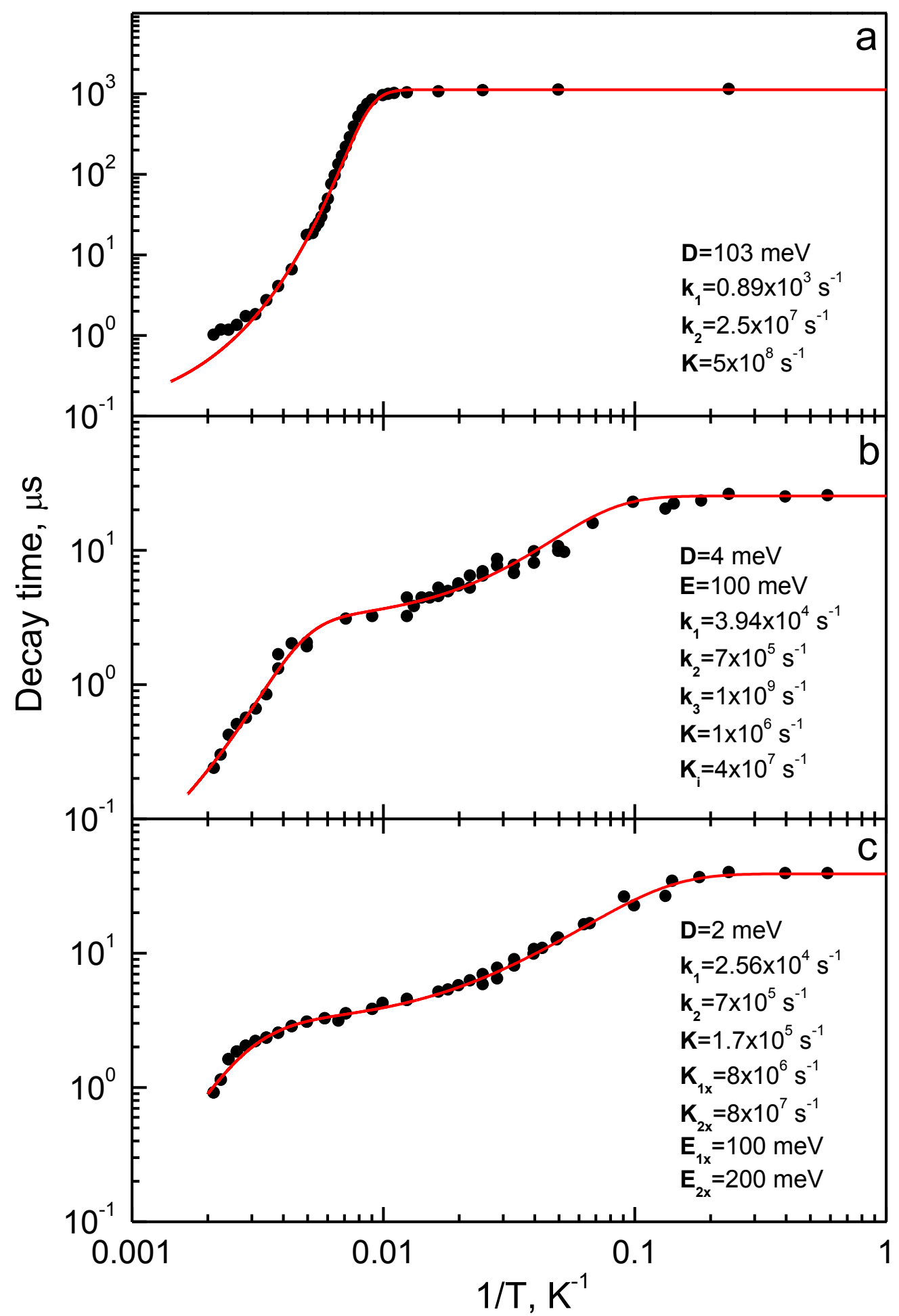

Fig. 1. Temperature dependences of the decay times of (a) the UV emission, (b) the $2.6 \mathrm{eV}$ emission, and (c) the $2.75 \mathrm{eV}$ emission of LuAG:Bi SCFs. Solid circles are experimental data. Solid lines are best fits of the two $(a, c)$ or three (b) excited-state level models shown in Fig. 4 to the experimental data, (for details see the text). The parameters of the fit are reported in the figures. (a) $\mathrm{E}_{\mathrm{exc}}=4.6 \mathrm{eV}, \mathrm{E}_{\mathrm{em}}=4.1 \mathrm{eV}$; (b) $\mathrm{E}_{\mathrm{exc}}=4.7 \mathrm{eV}, \mathrm{E}_{\mathrm{em}}=2.4 \mathrm{eV}$; (c) $\mathrm{E}_{\mathrm{exc}}=5.3 \mathrm{eV}, \mathrm{E}_{\mathrm{em}}=3.2$ $\mathrm{eV}$. 


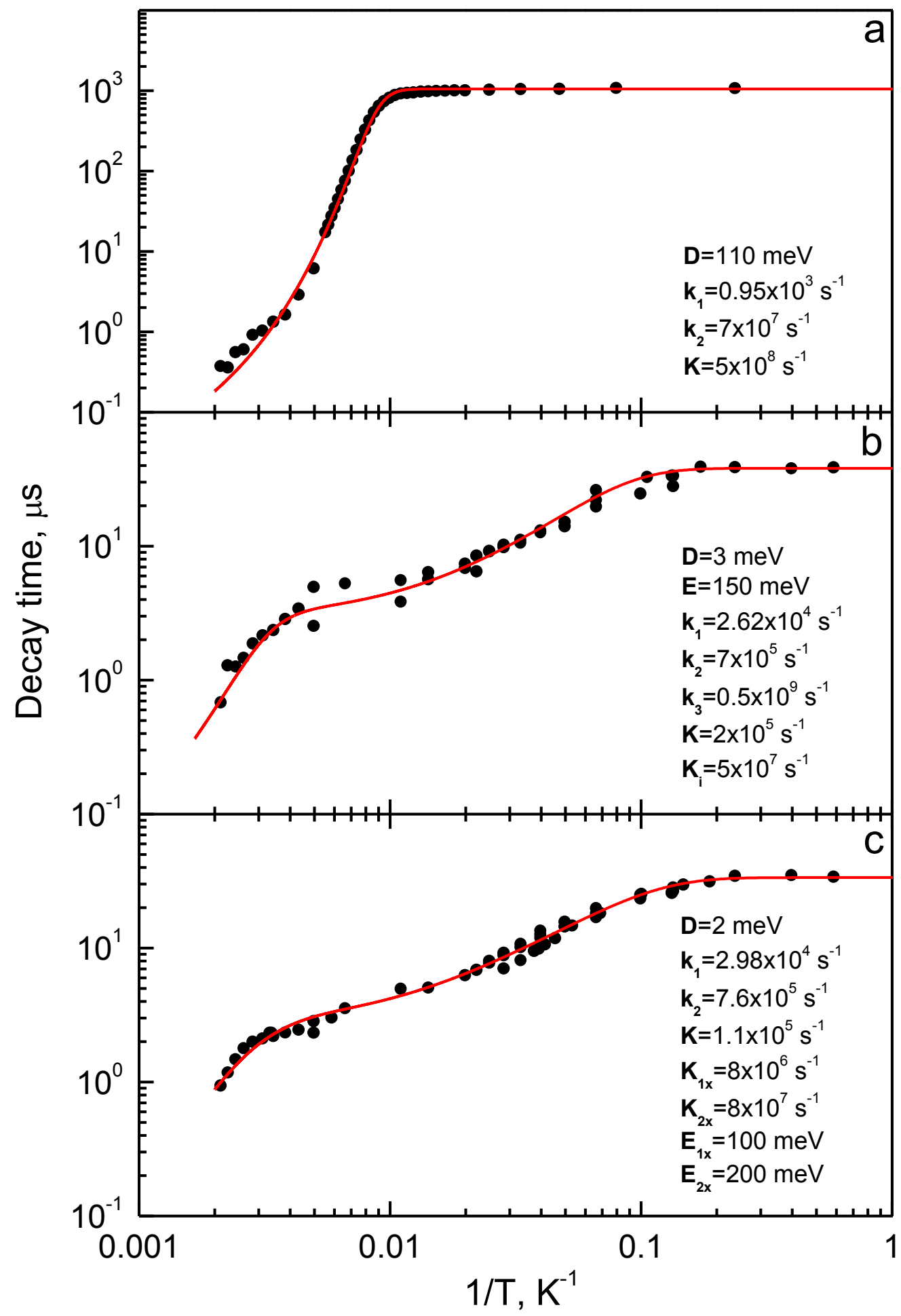

Fig. 2. Temperature dependences of the decay times of (a) the UV emission, (b) the 2.63 $\mathrm{eV}$ emission, and (c) the $2.75 \mathrm{eV}$ emission of YAG:Bi SCFs. Solid circles are experimental data. Solid lines are best fits of the two (a, c) or three (b) excited-state level models shown in Fig. 4 to the experimental data, (for details see the text). The parameters of the fit are reported in the figures. (a) $\mathrm{E}_{\mathrm{exc}}=4.55 \mathrm{eV}, \mathrm{E}_{\mathrm{em}}=4.0 \mathrm{eV}$; (b) $\mathrm{E}_{\mathrm{exc}}=4.5 \mathrm{eV}, \mathrm{E}_{\mathrm{em}}=2.4 \mathrm{eV}$; (c) $\mathrm{E}_{\mathrm{exc}}=5.3 \mathrm{eV}$, $\mathrm{E}_{\mathrm{em}}=3.1 \mathrm{eV}$. 


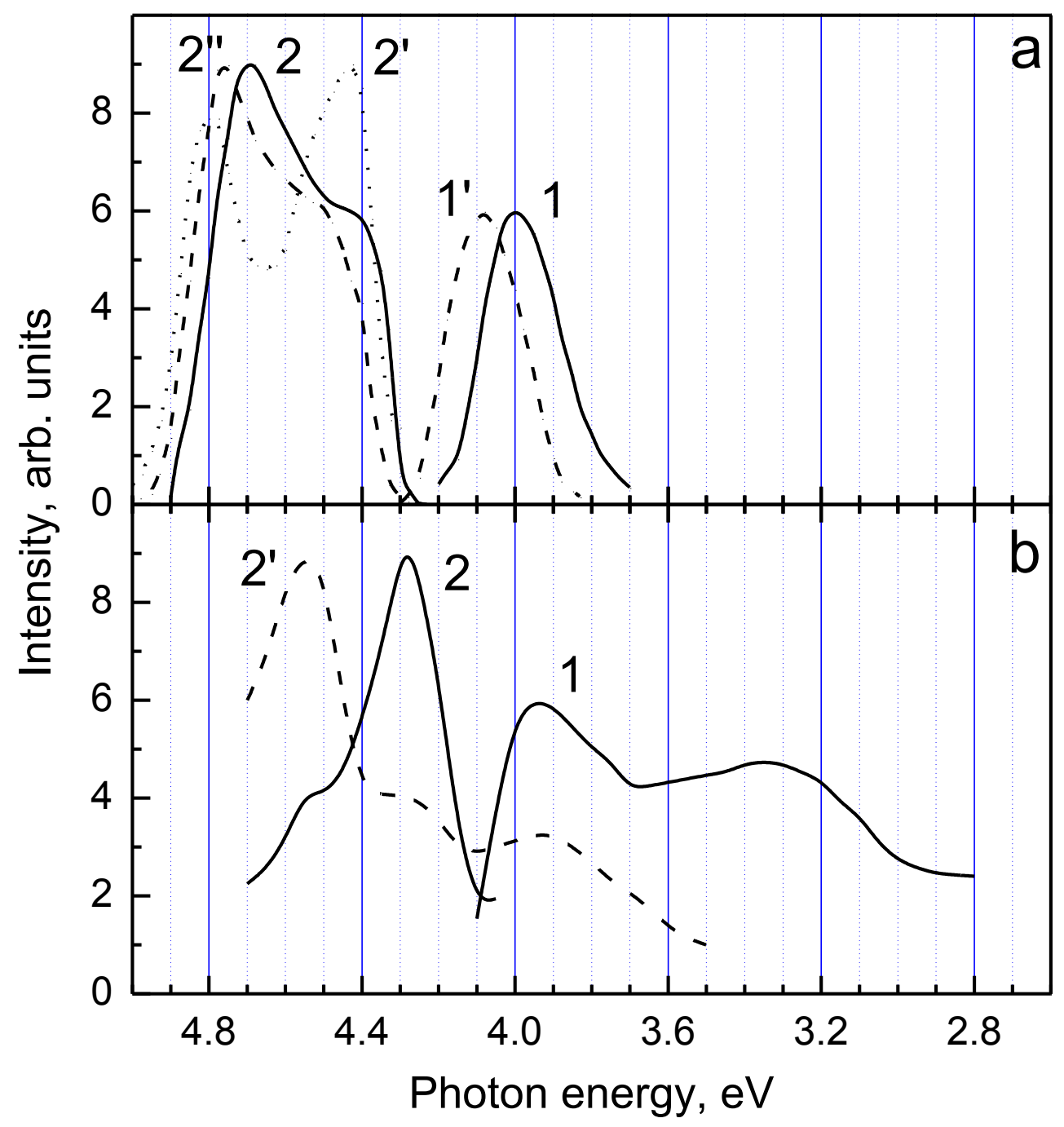

Fig. 3. (a) The UV emission bands (curves 1, 1') and the corresponding A excitation bands (curves 2, 2', 2') of $\mathrm{Bi}^{3+}$ centers in LuAG:Bi (curves 1, 2, 2') and YAG:Bi (curves 1', 2'). Curves 2 and 2' are measured at slightly different crystal positions. (b) Emission spectrum $\left(E_{\mathrm{exc}}=4.25 \mathrm{eV}\right.$ ) (curve 1) and excitation spectra for $\mathrm{E}_{\mathrm{em}}=3.85 \mathrm{eV}$ (curve 2) and $\mathrm{E}_{\mathrm{em}}=3.2 \mathrm{eV}$ (curve 2') measured for the YAG substrate. $\mathrm{T}=80 \mathrm{~K}$. 


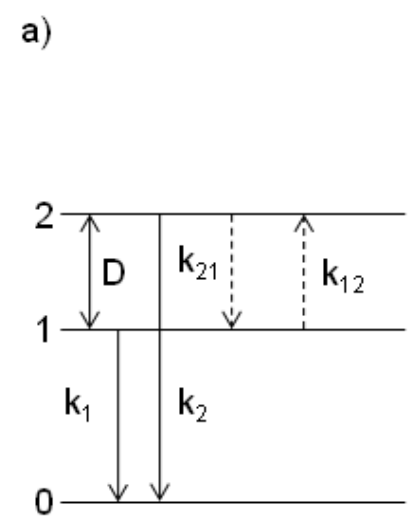

b)

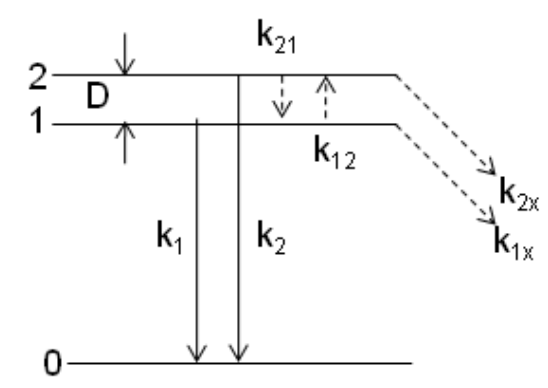

c)

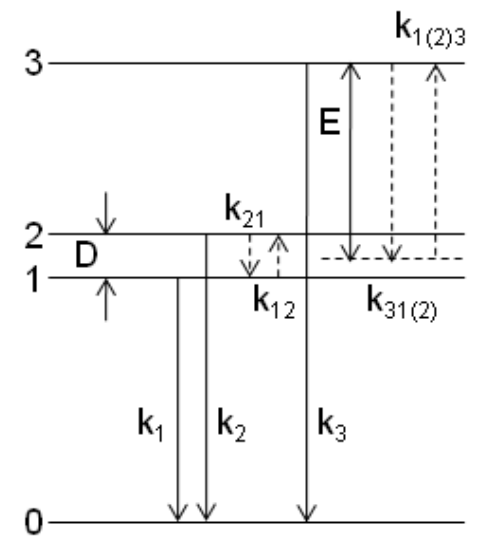

Fig. 4. Energy level diagrams used for the description of the excited states dynamics of the luminescence centers responsible for (a) the UV emission, (b) the $2.75 \mathrm{eV}$ emission, and (c) the the $\approx 2.6 \mathrm{eV}$ emission of $\mathrm{Bi}^{3+}$-doped aluminum garnets. For details, see the text. 


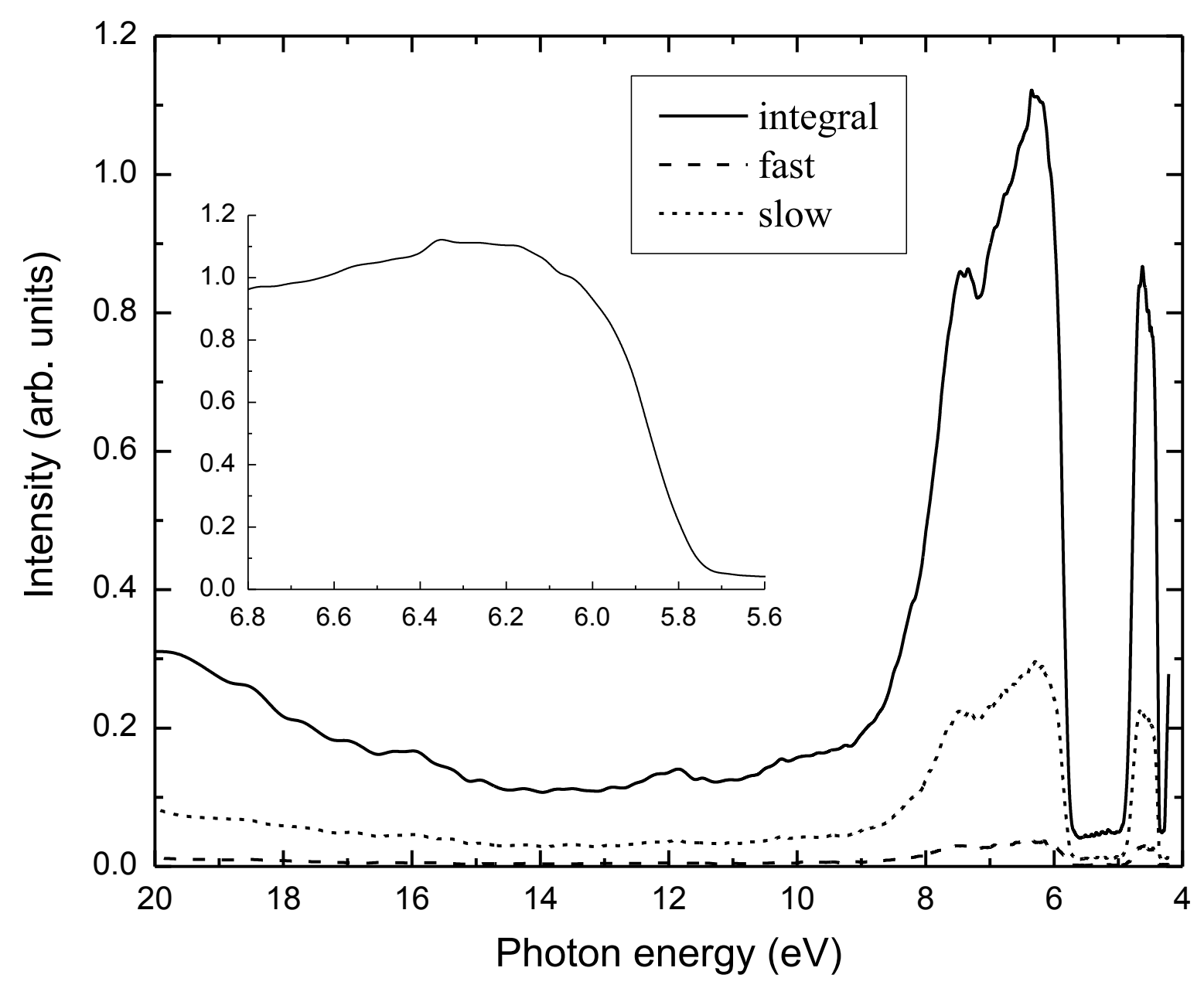

Fig. 5. Time-resolved excitation spectra measured at $10 \mathrm{~K}$ for the $\mathrm{UV}$ emission of $\mathrm{Bi}^{3+}$ centers in LuAG:Bi SCF. The time windows are 2-10 ns and 97-150 ns after the excitation pulse. In the inset, the integral spectrum in the 5.6-6.8 eV range is shown in more detail. 


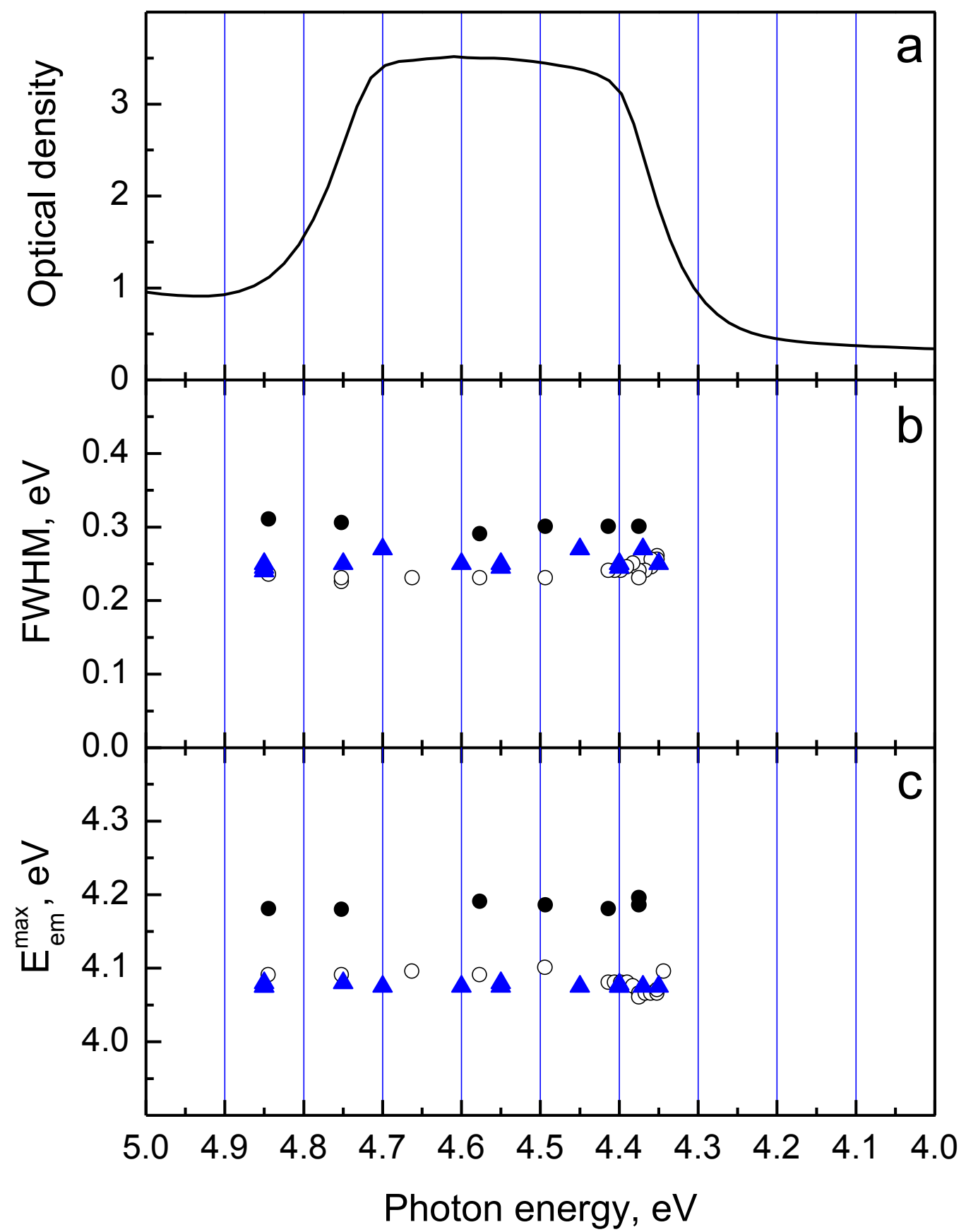

Fig. 6. (a) Absorption spectrum of LuAG:Bi SCF at 295 K. Dependences of the (b) FWHM and (c) maximum position of the UV emission band of LuAG:Bi SCF on the excitation energy measured at $4.2 \mathrm{~K}$ (empty circles), $80 \mathrm{~K}$ (triangles), and $295 \mathrm{~K}$ (solid circles). Spectral width of slit for the excitation was $5 \mathrm{~nm}$, and for the emission, $5 \mathrm{~nm}$ at $295 \mathrm{~K}$ and $3.5 \mathrm{~nm}$ at $4.2 \mathrm{~K}$. 


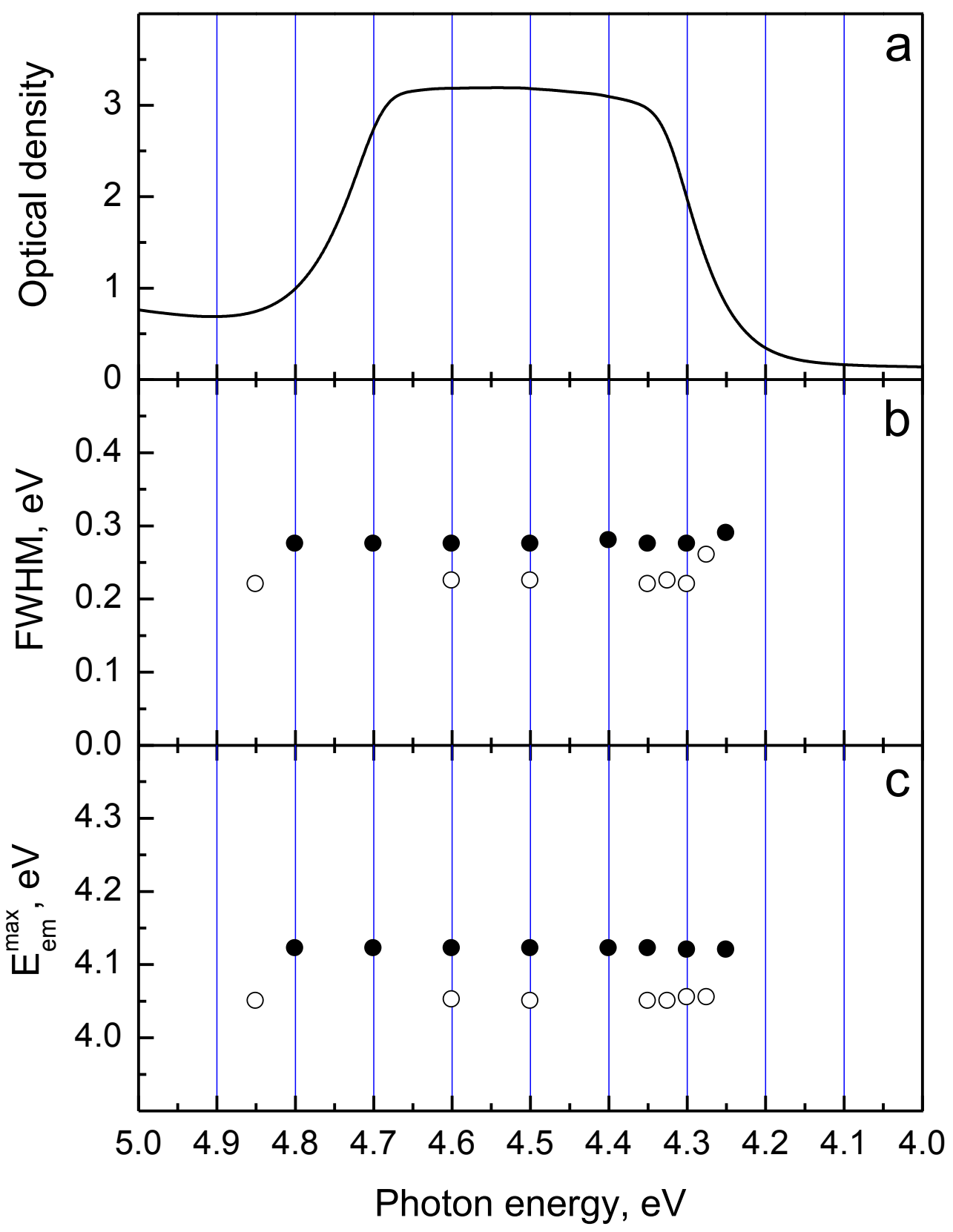

Fig. 7. (a) Absorption spectrum of YAG:Bi SCF at $295 \mathrm{~K}$. Dependences of the (b) FWHM and (c) maximum position of the UV emission band of YAG:Bi SCF on the excitation energy measured at $4.2 \mathrm{~K}$ (empty circles) and $295 \mathrm{~K}$ (solid circles). Spectral width of slit for the excitation was $5 \mathrm{~nm}$, and for the emission, $5 \mathrm{~nm}$ at $295 \mathrm{~K}$ and $3.5 \mathrm{~nm}$ at $4.2 \mathrm{~K}$. 


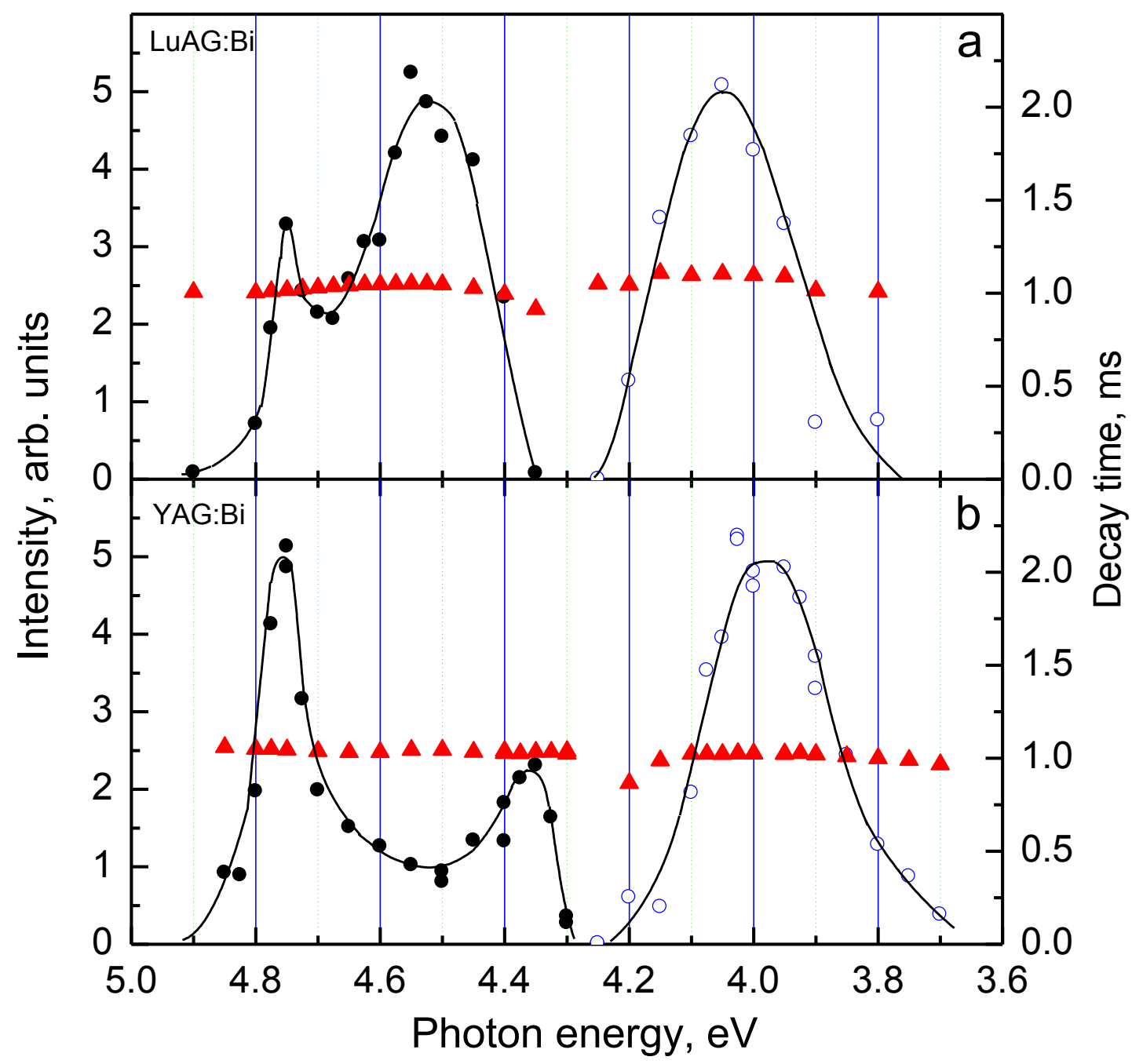

Fig. 8. Time-resolved UV emission (empty circles) and excitation (solid circles) spectra of (a) LuAG:Bi SCF and (b) YAG:Bi SCF and dependences of the decay time of the UV emission on the excitation energy and on the emission energy at $E_{\text {exc }}=4.35-4.4 \mathrm{eV}$ (triangles). $\mathrm{T}=4.2 \mathrm{~K}$. 